\title{
A STUDY ON A CASE OF SPINAL NEUROCYSTICERCOSIS: A RARE CASE REPORT
}

KEY WORDS: cysticercosis, intramedullary, spine

\section{Dr. Piyush Modi* MBBS PGJR 3 General Surgery GMC Haldwani. *Corresponding Author}

\section{Dr.prof. Rajeev} Kumar Singh

MBBS,MS,Professor General Surgery,GMC Haldwani

\section{Dr Prof. K.S Shahi MBBS,MS, professor General Surgery, GMC Almora}

\section{Dr Prateek Shakya MBBS,MS,Assistant professor General Surgery ,GMC Haldwani}

E $\quad$ Neurocysticercosis is the most common parasitic infection of the central nervous system worldwide. However, cysticercosis affecting the spine is considered extremely rare. We report one case of spinal cysticercosis with review of literature.

\section{INTRODUCTION}

Cysticercosis is the most common parasitic central nervous system ( CNS) infection worldwide.[1] The vast majority of neurocysticercosis are usually found at meningo-basal (30\%), parenchymal $(20 \%)$, intraventricular $(17 \%)$, intraspinal $(1 \%)$ or mixed locations (32\%). [2] intraspinal cases are extremely rare; incidence of spinal cysticercosis varies from $0.7 \%$ to $5.85 \%$. [3] spinal cysticercosis commonly affects the subarachnoid space compared to the spinal cord substance.[4]A purely intramedullary location as reported in this paper is quite exceptional . we report a cse of spinal cysticercois, diagnosed only after MRI report,

\section{CASE :}

A 23 yr old female Shivani presents with one month history of a gradually progressive weakness of both the lower limbs with associated pain and difficulty in walking for 1 month. Neurological examination disclosed decreased motor power in both the lower limbs.

MRI the bain was normal. MRI of the dorsolumbar spine was performed. Multiplaner conventional TlWl and T2Wl wererr acquired. Multiple large, oval shaped cystic lesion was seen involving the D12 to Ll vertebral level intraspinal intradural extramedullary indenting the spinal cord, homogenously hyperintense on T2W1 with slight peripheral edema.

The patient was treated conservatively with albendazole and steroids for 15 days follow up is being done showing recovery in the motor power of her lower limbs.

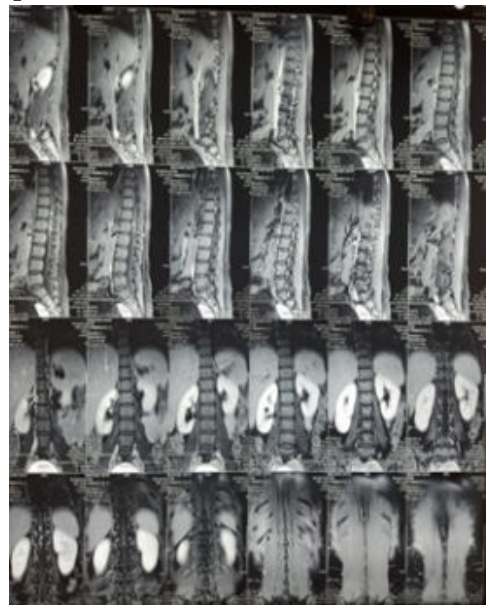

Figure 1 : magnetic resonance imaging saggital T2 weighted images showing a hyperintense lesion at D12 to Ll vertebral level of spine.

\section{DISCUSSION :}

Neurocysticercosis continues to be a major public health problem with a worldwide distribution showing a higher prevalence in the developing regions of the world.[5] However, prevalence is a variable depending mainly on sociocultural and economic factors. In addition, immigration from endemic to nonendemic areas is also one of the major factors influencing the prevalence.

CNS cysticercosis affects men and women equally. The peak incidence is between the third and fourth decades of life. It typically involves the brain parenchyma, intracranial. Spinal cysticercosis is rare, it may be leptomeningeal, intramedullary, or epidural. Among these, leptomeningeal is the most common, intramedullary is rare, and epidural is extremely rare.[2] Most cases of spinal cysticercosis are usually associated with cerebral cysticercosis. Isolated spinal cysticercosis either intamedullary or extramedullary is extremely rare.[6] In our case there was no evidence of either cerebral or systemic cysticercosis.

Clinical manifestation of spinal cysticercosis depend on the number and topography of the lesions, individual 's immune response to the parasite and the presence or absence of previous infestations. Clinically the most common manifestation of spinal neurocysticercosis is root pain and progressive weakness in contrast to the parenchymal neurocysticercosis that manifests with epileptic seizures; subarachnoid neurocysticercosis that manifests with headache while intraventricular neurocysticercosis manifests as subacute or intermittent syndrome of intracranial hypertension. Neurological damage in spinal intramedullary cysticercosis is attributed to the following factors; 1) mechanical compression caused by cyst, 2) due to the cord edema as a result of iinflammation caused by degenerating larva remnants and 3) gliosis. [7]

Sotelo and carpio had defined three clinical stages of neurocysticercosis, namely active, transitional and inactive neurocysticercosis. Escobar has also defined four pathological stages of neurocysticercosis. They are vesicular, colloidal vesicular, granular nodular, and nodular calcified. Vesicular is active form, colloidal vesicular and granular nodular represent transitional stages while nodular calcified stage is an inactive stage of neurocysticercosis.[8] Clinical suspicion of spinal cysticercosis is difficult, especially when there is neither previous history of any parasitic infestation nor associated cerebral neurocysticercosis, cerebrospinal fluid (CSF) examination should be done as it provides a reliable evidence of inflammation; further immunoblot test may be performed to confirm te diagnosis of neuro 
cysticercosis.[9] CSF examination was not done in our case; otherwise, it could have provided a provisional diagnosis. Apart from CSF studies, MRI is one of the most useful information in the evaluation of spinal neurocysticercosis patients. MRI in addition to the diagnosis also provides precise information about the disease activity and its location, carrying important therapeutic implications.[10] Mathuriya et al. had described MRI findings for the different stages of intramedullary cysticercosis; the pathognomonic diagnostic feature is the presence of cyst with an eccentric mural nodule representing the scolex showing a hypointense rim with hyerintense core on T2Wl and hypointense or isointense lesion on TIWl.[11] However these are not specific and differentials include: arachnnoid cyst, ependymal cyst, neurenteric cyst, sarcoidosis, ependymoma, and infections including abscess.[12] In cases of spinal cysticercosis, the entire neuraxis should be evaluated to detect any additional lesion. In contrast to the exixting literature, our case had isolated spinal cysticercosis.

A single therapeutic approach is not justifiable in spinal cysticercosis considering the pleomorphic nature of the disease. The decisive factors for the treatment of spinal cysticercosis are activity of the disease and location of parasites. Two mainstays of therapy are medical and surgical intervention. Surgical intervention should be considered in patients with acute onset of symptoms and in those where the diagnosis is in doubt as it not only provides decompression but also confirms the diagnosis after the histopathological examination and provides maximum chances of recovery avoiding the irreversible cord changes.[13] Results of surgical interventions are variable; mohanty et al. in case series of spinal intramedullary cysticercosis reported drastic recovery in 7/8 (87.5\%) cases.[14] However Sharma et al. reported improvement only in $60 \%$ cases.[15] Our case had received medical treatment, showing reasonably good recovery with minimal morbidity. Recent literature shows that according to American society for microbiology current consensus guidelines for treatment of neurocysticercosis, treatment of intramedullary / extramedullary spinal cysticercosis should be surgical.[16]

\section{CONCLUSIONS}

Spinal intramedullary cysticercosis remains a diagnostic challenge. It should be considered in the differential diagnosis of spinal intramedullary/ extramedullary lesions. Surgical treatment is recommended in patients with extensive lesions showing progressive neurological deficits. Medical treatment may be recommended in patients with early neurological symptoms under surveillance as surgical intervention may be required in case of neurological deterioration or failure of medical therapy.

\section{REFERENCES:}

1. Murthy JM, Raja Reddy D, Reddy PK. Intramedullary cysticercosis. Neurol India. 1988;36:316

2. Agarwal R, Chauhan SP, Mishra V, Singh PA, Gopal NN. Focal spinal intramedullary cysticercosis. Acta biomed. 2008;79:39-41.

3. Singh NN, Verma R, Pankaj BK, misra S. Cauda conus syndrome resulting from neurocysticercosis. Neurol India. 2003;51:118-20

4. Singh P, Sahai K. intramedullary cysticercosis. Neurol india .2004;52:264-5

5. Flisser A. Taeniasis and cysticercosis and to Taenia solium. In: Sun T, editor. Progress in clinical parasitology.Vol. 4.Florida:CRC Press; 1994.Pp.77-116

6. Kasliwal MK, Gupta DK, Suri V, Sharma BS, Garg A. Isolated spinal neurocysticercosis with clinical pleomorphism. Turk Neurosurg. 2008; 18: 294-7.

7. Holtzman RN, Hughes JE, Sachdev RK, Jarenwattananon A. Intramedullary cysticercosis. Surg Neurol. 1986;26:187-91.

8. Sotelo J, Marin C. Hydrocephalus secondary to cysticercotic arachnoiditis. A long-term foolow-up review of 92 cases.J Neurosurg. 1987;66:686-9.

9. Garcia HH, Gilman R, Martinez M, Tsang VC, Pilcher JB, Herrera G, et al. cysticercosis as a major cause of epilepsy in peru. The Cysticercosis Working Group in peru (CWG) Lancet. 1993;341:197-200.

10. Parmar H, Shah J, Patwardhan V, Patankar T, Patkar D, Muzumdar D, et al. MR imaging in intramedullary cysticercosis. Neuroradiology.2001;43:961-7.

11. Mathuriya SN, Khosla VK, Vasishta RK, Tewari MK, Pathak A, Prabhakar S. intramedullary cysticercosis:MRI diagnosis. Neurol India. 2001;49:71-4.

12. Qi B, Ge P,Yang H, Bi C, LiY, spinal intramedullary cysticercosis: a case report and literature review. Int J Med Sci. 2011;8:420-3.

13. Ahmad FU, Sharma BS. Treatment of intramedullary spinal cysticercosis: report of two cases and review of literature. Surg Neurol. 2007;67:74-7.

14. Mohanty A, Venkatrama SK, Das S. spinal intramedullary cysticercosis. Neurosurgery, 1997;40:82-7.
15. Sharma BS, Banerjee AK, Kak VK. Intramedullary spinal cysticercosis. Case report and review of literature. Clin Neurol Neurosur. 1987;89:1 1 1-6.

16. Garcia HH, Evans CA, Nash TE, Takayanagui OM, White AC, Jr, Botreo D, et al. current consensus guidelines for treatment of neurocysticercosis. Clin Microbiol Rev. 2001;15:747-56. 